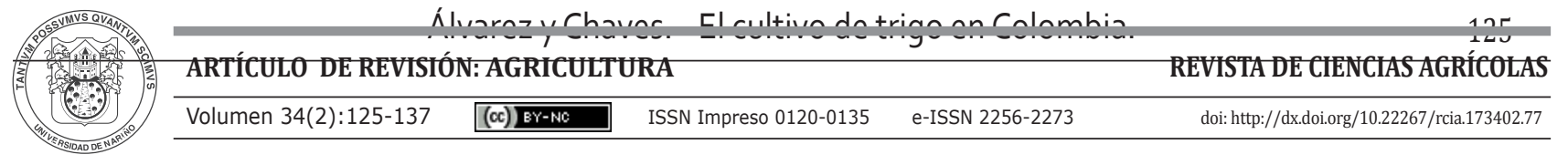

\title{
El cultivo de trigo en Colombia: Su agonía y posible desaparición
}

\author{
The wheat crop in Colombia: Its agony and possible disappearance
}

\section{David Álvarez Sánchez; Diana Melisa Chaves ${ }^{2}$}

1 M.Sc., Universidad de Nariño, Pasto, Colombia, daealvarezsa@unal.edu.co.

2 Ingeniera Agroindustrial, Universidad de Nariño, Pasto, Colombia, dmchavesm@udenar.edu.co.

Citar: Álvarez, D.; Chaves, D. 2017. El cultivo de trigo en Colombia: Su agonía y posible desaparición. Rev. Cienc. Agr. 34(2): 125 - 137. doi: http://dx.doi.org/10.22267/rcia.173402.77.

Recibido: Octubre 26 de $2016 . \quad$ Aceptado: Mayo 11 de 2017.

\section{RESUMEN}

El Trigo (Triticum aestivum L.) en Colombia es considerado un alimento básico además de requerir en su cadena productiva un número elevado de mano de obra, sin embargo, a nivel nacional desde el año de 1970 el área y producción del Trigo cultivado ha tenido una disminución constante representada en las estadísticas oficiales, siendo importante conocer las razones de dicho fenómeno y el impacto generado. Por lo anterior, esta revisión buscó identificar los aspectos nocivos que explican la agonía y posible desaparición de este sistema productivo en el país en tres periodos históricos: i) desde 1920 hasta 1989, ii) desde 1990 hasta 2000 y iii) perspectivas a futuro. Para ello, se recopiló información de diferentes fuentes primarias y secundarias, concluyendo que el Trigo a lo largo del tiempo ha soportado coyunturas macroeconómicas y el desamparo gubernamental en el sector, poniendo en duda la sustentabilidad del cultivo en Colombia.

Palabras clave: Triticum aestivum L., Tratado Libre Comercio (TLC), harina de trigo, cereal. 


\begin{abstract}
Wheat (Triticum aestivum L.) in Colombia, which is requires a large workforce in its productive chain, is considered a staple food of the national diet, however, since 1970 the area and production of the cultivated wheat has had a constant decrease represented in the official statistics, being important to know the reasons for this phenomenon and the impact generated. Accordingly, this review sought to identify the harmful aspects that explain the agony and possible crop's disappearance in the country in three historical periods: i) from 1920 to 1989, ii) from 1990 to 2000 and iii) future perspectives. Information was collected from different primary and secondary sources, it was concluded that the wheat has witnessed different macroeconomics downturns which has led to the government's abandonment in the sector. This in turn has raised many questions regarding the crop's sustainability in Colombia.
\end{abstract}

Key word: Triticum aestivum L., Free Trade Agreement (FTA), wheat flour, cereal.

\section{INTRODUCCIÓN}

Estudios con marcadores moleculares han demostrado que la domesticación del Trigo (Triticum aestivum L.) inició alrededor del año $12.000 \mathrm{AC}$, en las montañas del sureste de Turquía en donde este cereal tiene su origen; 2.000 años más tarde el trigo se distribuyó hacia el norte y el sur de Mesopotamia por la cuenca mediterránea hasta llegar a Italia y España en el año 7.000 A. C. (Mac Key, 2005).

Cristóbal Colón, durante su segundo viaje el 25 de septiembre de 1493 desde España, transportó las primeras semillas de trigo a las Américas, más tarde, Jerónimo Lebrón de Quiñones entre 1530 y 1535 introduciría el Trigo junto con la Avena y la Cebada al Nuevo Reino de Granada (Colombia), llegando inicialmente por el puerto de Santa Marta (Patiño, 1969, FENALCE, 2010).

El trigo traído y conservado por el español hace parte indispensable de su dieta alimenticia que no fue aceptada por el indígena (Trujillo et al.,1990) y aunque el español sí asimiló el Maíz, nunca abandonó el consumo de pan de Trigo. Quizás por esta razón, no solo fue este cereal, una de las primeras semillas en cultivarse y procesarse en tierras americanas, sino que conservó con mayor fidelidad el proceso de producción que por entonces se daba en España.

En 1540 se instalan los primeros molinos en las zonas altas de Nariño y Boyacá (Trujillo et al., 1990). Desde la época colonial hasta hoy en día, el trigo en su calidad de alimento básico es parte importante para la sociedad urbana y rural (Yepes, 1948); siendo de gran relevancia principalmente por su alto valor energético y las numerosas formas de procesamiento, considerándose la harina de trigo y los productos procesados a partir de ella, básicos en la canasta familiar.

Valderrama (1976) describe que en Colombia se distinguen especialmente dos zonas productoras de Trigo: la del departamento de Nariño y la de los departamentos de Cundinamarca y Boyacá, donde la franja triguera está localizada por encima de los 2.200 hasta los 3.000 msnm. Esta descripción permanece estática en el tiempo ya que recrea la distribución reportada en el 2016 por la Federación Nacional de Cultivadores de 
Cereales y Leguminosas (FENALCE, 2017) y por el Ministerio de Agricultura y Desarrollo Rural (MADR, 2017).

Para el año de 1950 en Colombia se sembraron cerca de 145.000ha de Trigo, cosechando 102.000t (Valderrama, 1976). En el 2016, solamente se reportaron 6.195ha sembradas y una producción de 13.476t de trigo (FENALCE, 2017). La tasa de crecimiento anual en área según MADR (2017) es del -9,0\% analizando los valores desde 1986 al 2013. Esto indica un decrecimiento que puede llevar a la desaparición del cultivo en los próximos años.

En este documento, se analizó el comportamiento de Trigo en Colombia a lo largo de tres momentos históricos decisivos para este cereal: i) desde 1920 hasta 1989, ii) desde 1990 hasta 2000 y iii) 2001 y perspectivas a futuro, reflexionando sobre los principales factores que incidieron sobre la producción y que han originado la agonía y posible desaparición de este renglón productivo.

\section{Trigo 1920 - 1989}

La era dorada del Trigo por su expansión y desarrollo puede ubicarse entre 1920 y 1960 , donde se iniciaron los primeros trabajos científicos organizados y se consolidaron diferentes centros de investigación en el país financiados por capital estatal. Muestra de ello, es la creación de la primera granja destinada a la investigación en cereales y cultivos de clima frio "La Picota", ubicada en el departamento de Cundinamarca e inaugurada en 1925. Más tarde se anexarían las granjas "La Isla" y "Tibaitatá" (Cundinamarca), "Bonza” (Boyacá), "Obonuco" (Nariño) y la "Granja experimental de Palmira" (Valle del Cauca) (Zarama, 1999).

Se iniciaría con ello, el proceso de mejoramiento genético de Trigo por parte del Departamento de Investigaciones Agropecuarias (DIA) en alianza con la Fundación Rockefeller. El principal objetivo de este programa fue el desarrollo de variedades resistentes, sea a la roya del tallo (Puccinia graminis f.sp. tritici) para los Trigos sembrados en áreas más cálidas, o bien a la roya amarilla (Puccinia striiformis West.) para las zonas más templadas (IICA, 1967).

Se destaca en este periodo las variedades Picota, Bola y Diacol Nariño; en 1952, se liberaría la primera variedad adaptada y mejorada Menkemen 50, como un esfuerzo para contrarrestar la presión de la roya como principal limitante del cultivo (Adams y Mancini, 1964) (Tabla 1).

Tabla 1. Algunas variedades liberadas entre 1945 y 1960 por el Departamento de Investigaciones Agropecuarias de Colombia.

\begin{tabular}{cl}
\hline Variedad & \multicolumn{1}{c}{ Características } \\
\hline Menkemen 50 & Resistente a la roya de la hoja, con un rendimiento entre los 1,8-2,7 t.ha ${ }^{-1}$ \\
\hline \multirow{2}{*}{ Picota } & $\begin{array}{l}\text { Resistente al volcamiento, espiga roja y mutica larga; grano cilíndrico de color blanco } \\
\text { crema, consistencia semidura. Rendimiento 4,0 t.ha }{ }^{-1} \text {. }\end{array}$ \\
\hline \multirow{2}{*}{ Bola } & $\begin{array}{l}\text { Altura más de } 1,5 \mathrm{~m}, \text { sensible al volcamiento. Rendimiento 3,0 t.ha }{ }^{-1} \text {. Adaptación } \\
2.500 \text { a } 3.000 \mathrm{msnm} .\end{array}$ \\
\hline
\end{tabular}

Fuente: Tapia (1940). 
Entre 1960 y 1990 se liberaría por parte del Programa de Cereales más de 12 variedades adaptadas a las zonas productoras de Colombia, con buen rendimiento y principalmente con tolerancia o resistencia a las diferentes royas (Tabla 2). Esto incrementó el rendimiento por hectárea y otorgó al agricultor una oferta varietal amplia. De esta forma, el trigo en Colombia fue estimulado desde los centros de investigación.

En 1962 se produjo la primera variedad multilineal distribuida comercialmente en el mundo (Castro, 1980). Paralelamente, a lo largo del país se realizarían diferentes estu- dios de aptitud agroindustrial para las nuevas variedades.

En 1972, el gobierno Colombiano intensificó el programa de producción de Trigo, ahora liderado por el Instituto Colombiano Agropecuario ICA (el cual remplazó al DIA), buscando variedades con altos rendimientos, buena calidad molinera y panadera, amplia adaptación, con resistencia al vuelco, tolerancia a las enfermedades y además, precoces. Veinte nuevas variedades fueron plantadas y evaluadas en 33 localidades desde los 2.300 a los $3.000 \mathrm{msnm}$ durante los años 1972 a 1974 (Zapata et al., 1974).

Tabla 2. Algunas variedades liberadas entre 1961 y 1990 por el Instituto Colombiano Agropecuario de Colombia.

\begin{tabular}{|c|c|c|}
\hline Variedad & Características & Fuente \\
\hline Bonsa & $\begin{array}{l}\text { Rendimiento aproximado de } 2,4-2,9 \text { t.ha }{ }^{-1} \text {, moderadamente } \\
\text { susceptible a roya amarilla y susceptible a roya del tallo, recomendad } \\
\text { para zonas entre los } 2.400 \text { y } 2.800 \mathrm{msnm}\end{array}$ & \multirow{2}{*}{$\begin{array}{l}\text { (Zapata y López, } \\
\text { 1967) }\end{array}$} \\
\hline Crespo & $\begin{array}{l}\text { Resistente a la roya amarillay relativamente sensible al vaneamiento, } \\
\text { tiene rendimiento de } 2,1-3,2 \text { t.ha }^{-1} \text {. } \\
\text { Recomendada para zonas entre los } 2.400 \text { y } 2.800 \text { msnm. }\end{array}$ & \\
\hline Tota & $\begin{array}{l}\text { Recomendada para zonas localizadas entre los } 2.200 \text { y } 2.700 \mathrm{msnm} \text {. } \\
\text { Su cosecha es precoz, tiene un rendimiento de } 1,4-1,7 \text { t.ha }^{-1}\end{array}$ & $\begin{array}{l}\text { (Rincon et al., } \\
\text { 1981) }\end{array}$ \\
\hline Tiba & $\begin{array}{l}\text { Resistente al vaneamiento, moderadamente resistente a la roya } \\
\text { amarilla, recomendable para zonas entre los } 2.200 \text { y } 2.700 \text { msnm. } \\
\text { Rendimiento aproximado de } 2,7 \mathrm{t}^{-h^{-2}}\end{array}$ & \multirow{3}{*}{$\begin{array}{l}\text { (Zapata y López, } \\
\text { 1967) }\end{array}$} \\
\hline Napo & $\begin{array}{l}\text { Resistente a roya amarilla y al vaneamiento, su rendimiento } \\
\text { aproximado es de } 3095 \mathrm{~kg} / \mathrm{ha} \text {, se recomienda para zonas entre los } \\
2.500 \text { y } 3.000 \mathrm{msnm}\end{array}$ & \\
\hline Miramar & $\begin{array}{l}\text { Variedad multilineal resultado de una mezcla de } 10 \text { líneas similares, } \\
\text { altamente resistente a roya amarilla y al vaneamiento, }\end{array}$ & \\
\hline Yuriya & $\begin{array}{l}\text { Recomendad para alturas entre } 2.200 \text { y } 2.700 \mathrm{msnm} \text {, con un ciclo } \\
\text { de vida de } 150 \text { días y un rendimiento de } 3 \text { t.ha }^{-1}\end{array}$ & (Castro, 1980) \\
\hline Sugamuxi & 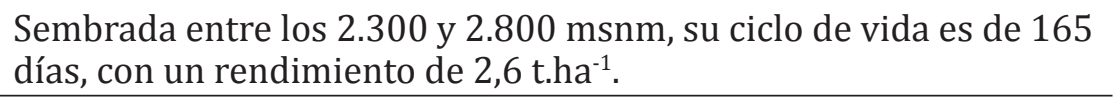 & \multirow{2}{*}{$\begin{array}{l}\text { (Rincon et al., } \\
\text { 1981) }\end{array}$} \\
\hline Samaca & $\begin{array}{l}\text { Moderadamente susceptible a roya amarilla y moderadamente } \\
\text { resistente a roya del tallo, con un rendimiento de } 2,5-3,2 \text { t.ha }^{-1}\end{array}$ & \\
\hline
\end{tabular}


Se destacan también las acciones de la Caja Agraria, entidad que suministraría la mayor parte de las semillas mejoradas, fertilizantes y créditos para los agricultores, incentivando la siembra de Trigo en Colombia (Adams y Mancini, 1964). El Programa de trigo con la liberación de los nuevos materiales de 1950 a 1973 aumentó el promedio nacional de 0,8 a 1,3 t.ha ${ }^{-1}$ (Zapata et al., 1974). Sin embargo, y a pesar de los grandes avances en el país, como lo indica Valderrama (1976), a partir de 1970 se disminuirían los recursos destinados al Programa de Cereales, así como el número de investigaciones de Trigo en Colombia.

Factores de desaceleración del Trigo. La historia del Trigo en Colombia ha estado relacionada directa o indirectamente con Estados Unidos y el diferencial en la producción que existe entre los dos países. En 1930, debido a los subsidios e incentivos, así como al alto grado de industrialización de la agricultura, la producción de granos en el país norteamericano comenzó a exceder ampliamente la demanda interna. Esta sobreproducción se desarrolló especialmente durante la "Guerra Fría" periodo en el cual se aprueba la ley pública 480 de 1954, mediante la cual se aprobaron grandes donaciones de trigo y otros cereales a países subdesarrollados. Estados Unidos utilizó las donaciones para ganar aliados y al mismo tiempo se deshizo de la sobreproducción agrícola (Bejarano, 1989; Afanador, 2008).

La anterior medida tuvo un impacto profundo sobre la producción agrícola, la alimentación y la economía de los países receptores, entre ellos Colombia. Desde 1946, el índice de consumo de trigo en el país se incrementó a 14 kilos por habitante, reemplazando parte de los productos tradicionales como el maíz, y creando la necesidad de importar trigo para satisfacer la nueva tendencia de consumo interno (Yepes, 1948, Adams y Mancini, 1964). Afanador (2008) reporta que el trigo importado pasó de $22 \%$ en 1950 a cerca del $90 \%$ en 1971. En 1973, el país tiene un consumo de 480.000 toneladas de las cuales solo se produjeron 60.000 e importaron 420.000 toneladas por un valor de 71,4 millones de dólares (U.S. 173, valor CIF de una tonelada) (Zapata et al., 1974). La harina de trigo y el trigo representaron en promedio, el $56 \%$ del valor de todos los productos importados desde Estados Unidos (Adams y Mancini, 1964; IICA, 1967; Zapata et al., 1974).

Entre 1950 y 1974 la tasa de producción de trigo en Colombia presentó una disminución de $-3,6 \%$ por año, el cual es uno de los más altos en la región (Valderrama, 1976). Para esta época, la mayoría de los cultivos se concentraban en las tierras altas de clima frío, donde el trigo competía con la cebada, la papa, el maíz y la producción de leche; Cundinamarca suministraba un promedio de 30 al $40 \%$ del total de la producción nacional, Boyacá cerca de un 30\% y Nariño del 20 al 30\% (Adams y Mancini, 1964). La progresiva disminución en el área sembrada ocasionaría desequilibrios en los modos de ingreso familiar en estas regiones, estadísticas del Departamento Administrativo Nacional de Estadística (DANE, 1974) indican que, en promedio, cada hectárea cultivada empleaba 28 jornales.

En 1974, se sembró solo el 31\% de lo sembrado en 1950, y el 25\% con respecto a 1955 (Trujillo et al, 1990). Aunque la producción ha descendido, este fenómeno no es tan marcado debido a los aumentos en la productividad, enmarcada en el contexto mundial de la Revolución Verde, donde el cultivo de trigo pasó de producir 0,68 t.ha $^{-1}$ en 1950 a 1,6 t.ha $^{-1}$ en 1986 (Adams y Mancini, 1964; MADR, 2017). 
Según Valderrama (1976), el gobierno colombiano busca proteger al sector triguero ubicándolo dentro de la política de producción de alimentos, eliminando los subsidios a las importaciones y aumentando los precios internos de sustentación. Sin embargo, el trigo proveniente de Estados Unidos no desciende y se crea entonces un primer panorama de conflicto de rentabilidad para los pequeños y medianos productores de Colombia.

\section{Trigo, 1990 - 2000}

En la década de los 90 se dio inicio a la apertura económica, la cual condujo a una transformación en la economía nacional. En su afán de internacionalización, el gobierno colombiano llevó a la nación a una de las peores crisis de su historia, afectando sectores como el industrial y el agrícola principalmente (Londoño, 1998; Burbano y Ortega, 2015).

Los agricultores que sembraban trigo habían tolerado con esfuerzo las importaciones que se realizaban hasta la fecha en Colombia, ahora, según Zarama (1999) serían doblemente afectados. La producción de trigo disminuyó en una proporción del 60 al 70\% en el País (Moreno, 2007; Viloria, 2007), por ejemplo, la industria de la galletería había incrementado paulatinamente el uso de harina de trigo importada, la cual presenta características deseables para esta industria, reduciendo la demanda de trigos regionales.

El sector industrial harinero se vio favorecido y afectado al mismo tiempo, pues si bien el trigo extranjero abundaba, el precio aún no era estable, además, la competencia de harinas importadas era cada día más agresiva. En 1995, varios molinos cerraron operaciones y otros sufrieron cuantiosas pérdidas. Para 1997, en el país quedaban 16 empresas mo- lineras, las cuales controlaban el $86 \%$ de las compras totales de trigo (Ripoll, 2014).

Con un modelo económico abierto, el país confió en el Instituto Nacional de Abastecimiento (INA) más tarde IDEMA (Instituto de Mercadeo Agropecuario), el cual regía como la entidad encargada de intervenir y controlar las importaciones de materias primas como el trigo. Además, el IDEMA se encargaba de distribuir el cereal a los molinos del país, el proceso de regulación se basaba en compras y distribución a la industria procesadora, adquiriendo del 20 al 30\% del trigo nacional. Para los empresarios, el trigo colombiano incrementaba sus costos debido al precio del mismo y la supuesta baja calidad (Ripoll, 2014). Una de las causas demandadas es el porcentaje de humedad que el cereal tiene al momento de su comercialización y la deficiente clasificación del grano de acuerdo con el tamaño.

El gremio productor de trigo, cebada, sorgo y soya, principales afectados por la apertura económica, establecieron convenios de transición en el precio de venta con el sector agroindustrial para amortiguar los efectos negativos de la política económica. Paralelamente, el gobierno nacional suscribe en Cartagena la decisión 371 del Grupo Andino, con el objeto de estabilizar el costo de importación de materias primas creando el Sistema Andino de Franjas de Precios (SAFP).

Sumado a lo anterior, el 11 de noviembre de 1994 se aprueba el decreto 2524, en el cual se establecen las reglas para la fijación de cuotas de absorción de materias primas de producción nacional por parte de la industria y condiciona el establecimiento de vistos buenos de importación al cumplimiento de los convenios de absorción. Para muchos, la industria 
harinera debía adquirir volúmenes de cosechas cada vez mayores y más costosos, que posteriormente los consumidores pagarían con una mayor inflación; sin embargo, esta medida de protección agrícola le brindaría a los productores de trigo un descanso e impulso económico.

Dentro de los factores que incidieron en la baja rentabilidad del trigo para este periodo, se encuentran principalmente las malas condiciones de transporte desde el campo hasta las zonas de procesamiento (Burbano y Ortega, 2015), un incremento atípico en el precio del dólar (hasta del 130\%) que se reflejó en los costos de los insumos agrícolas, y la liquidación del IDEMA en 1997 por la falta de sos- tenibilidad financiera, descuido del gobierno nacional y la presión del gremio de molineros que consideraban que esta entidad obstaculizaba el crecimiento y modernización del sector harinero (Machado, 2009; Ripoll, 2014).

El seguimiento cronológico de la historia del trigo en Colombia, revela que no se puede desconocer que la agricultura se encuentra estrechamente relacionada con los problemas de orden público, pues de 1994 a 2002 se presenta un momento crítico en el país que repercute sobre la economía, dado que la inseguridad e incertidumbre llevaron a que las inversiones en la industria y la agricultura se redujeran, castigando la pequeña demanda de trigo nacional existente.

Tabla 3. Algunas variedades liberadas entre 1991 y 2000 por el Instituto Colombiano Agropecuario de Colombia.

\begin{tabular}{|c|c|c|}
\hline Variedad & Características & Fuente \\
\hline Tenza & $\begin{array}{l}\text { Altura de la planta de } 108 \mathrm{~cm} \text {, su espigamiento y cosecha alrededor } \\
\text { de los } 170 \text { días, es susceptible al volcamiento. }\end{array}$ & $\begin{array}{l}\text { (Castro et al., } \\
1991)\end{array}$ \\
\hline Hunza & $\begin{array}{l}\text { Sembrada en zonas entre los } 2.400 \text { y } 2.800 \mathrm{msnm} \text {, resistente a la } \\
\text { roya amarilla y de la hoja. }\end{array}$ & $\begin{array}{l}\text { (Brito et al., } \\
\text { 1993) }\end{array}$ \\
\hline Bochica & $\begin{array}{l}\text { Resistente a la roya amarilla, con un rendimiento aproximado de } \\
3,5-4,2 \text { t.ha }^{-1}\end{array}$ & $\begin{array}{l}\text { (Brito et al., } \\
\text { 1991) }\end{array}$ \\
\hline Yacuanquer & $\begin{array}{l}\text { Rendimiento de } 3,5 \text { t.ha }^{-1} \text {, resistente a la roya amarilla, negra y } \\
\text { parda y al volcamiento pero susceptible a enanismo amarillo, su } \\
\text { periodo entre los } 178 \text { a } 179 \text { días. }\end{array}$ & $\begin{array}{l}\text { (Amaguaña, et al., } \\
\text { 1995) }\end{array}$ \\
\hline Gualmatan & $\begin{array}{l}\text { Rendimiento de } 1,7-2,3 \text { t.ha }^{-1} \text {, variedad recomendada para zonas } \\
\text { entre } 2.000 \text { y } 3.000 \mathrm{msnm} .\end{array}$ & $\begin{array}{l}\text { (Bolaños et al., } \\
\text { 1991) }\end{array}$ \\
\hline Achalay & $\begin{array}{l}\text { Recomendada para Nariño en zonas por encima de los } 2.400 \\
\text { msnm, presenta resistencia moderada al enanismo y a la roya } \\
\text { parda, negra y amarilla, tiene un rendimiento aproximado de } 3,2 \\
\text { t.ha-1. }\end{array}$ & $\begin{array}{l}\text { (Bolaños et al., } \\
\text { 1993) }\end{array}$ \\
\hline $\begin{array}{l}\text { Obonuco } \\
\text { Sequia } 96\end{array}$ & $\begin{array}{l}\text { Resistente a manchas foliares, pudrición de granos y raíces, y } \\
\text { resistencia moderada a la roya amarilla, con un rendimiento } \\
\text { promedio de } 2 \text { t.ha }^{-1} \text {. }\end{array}$ & $\begin{array}{l}\text { (Merchancano y } \\
\text { Bolaños 1996) }\end{array}$ \\
\hline $\begin{array}{l}\text { Obonuco } \\
\text { Sureño } 97\end{array}$ & $\begin{array}{l}\text { Rendimiento de } 3,2 \text { t.ha }^{-1} \text {, Presenta resistencia a la roya amarilla y } \\
\text { a la roya del tallo y de la hoja, y resistencia moderada al enanismo, } \\
\text { y pudrición de las hojas. }\end{array}$ & $\begin{array}{l}\text { (Bolaños et } \\
\text { al.,1997) }\end{array}$ \\
\hline
\end{tabular}


Nuevamente, la academia, los centros de investigación y los gremios de agricultores, soportan el cultivo de trigo con trabajos de extensión, investigación y desarrollo de variedades mejoradas (Tabla 3); buscaron sostener esta actividad en contra de un panorama difícil. Brindar al agricultor materiales genéticos que tengan un mejor rendimiento y soporten factores bióticos y abióticos, se convierte en una propuesta para este sector agrícola.

\section{El Trigo, desde 2001 y perspectivas a futuro}

En el 2005, la importación de trigo a nivel nacional ascendió a 1,6 millones de toneladas, de las cuales el $66 \%$ se importó de Estados Unidos, el 26\% de Canadá y el 8\% de Argentina. En ese mismo año, la producción nacional correspondió a menos del 5\% de las importaciones (Espinal et al., 2006, Ripoll, 2014). Bajo este panorama, el departamento de Nariño participó con el $60 \%$ de la producción nacional, mostrando el rendimiento promedio más alto de Colombia. De todas formas, la producción nacional y los rendimientos son marginales al compararlos con países templados como Estados Unidos o Canadá (Espinal et al., 2006, Ripoll, 2014).

En el estudio socioeconómico realizado por Pareja y Méndez (2006) se describe un cultivo de trigo que se desarrolla aún de forma tradicional, en condiciones de minifundismo y con alta demanda de mano de obra familiar. En promedio para el 2006, el cultivo generó 12.352 jornales (14 jornales.ha-1 ${ }^{-1}$, sumado a 667 puestos de trabajo en la cadena de producción, con un promedio de dos puestos por hectárea sembrada (DANE, 2011; MADR, 2017). El cultivo de trigo como generador de ingreso se ve nuevamente amenazado, esta vez por uno de los grandes sucesos ocurridos en el siglo XXI para la agricultura en Colombia, los Tratados de Libre Comercio (TLC).

El gobierno busca a través de los TLC, la reducción de aranceles para aumentar las importaciones y las exportaciones de diferentes productos, y de esta manera, fortalecer la economía nacional. El TLC con Estados Unidos, se firmó el 4 de julio de 2007, y un año más tarde el TLC con Canadá, el 21 de noviembre de 2008; la vigencia del primero se instauró en mayo de 2012, y del segundo en agosto de 2011 (MCIT et al., 2012; Burbano y Ortega, 2015). Los anteriores acuerdos internacionales redistribuirían las importaciones de trigo en Colombia, en donde el $48 \%$ sería importado por Canadá, el $22 \%$ por Estados Unidos, el 22\% por Argentina y el 8\% por Paraguay (Ripoll, 2014).

Como se ha venido indicando, históricamente el productor de trigo se enfrenta a desigualdades comerciales globales, y con la vigencia de los TLC, se compite con agricultores que para ese momento reciben subsidios 16 veces mayores a los otorgados al agricultor nacional, esto evidencia una clara inequidad y desconocimiento del sector agropecuario al momento de firmar los tratados (Merheg, 2005). El panorama se agravó cuando el trigo y la cebada fueron agrupados en la categorización Canasta A9, es decir, sin protección desde el primer día de vigencia del tratado (MCIT et al., 2012; Burbano y Ortega, 2015).

El TLC permitió que Estados Unidos continuara con la política de subsidios a la producción interna, mientras se solicita a Colombia el desmonte del Sistema Andino de Franjas de Precios. Cultivos como el maíz, la soja y el trigo tuvieron un impacto calculado en la disminución del $20 \%$ en el valor anual de la producción (Nuñez et al., 2013). Para dimensionar la desigualdad en términos de volumen de cereal producido, Estados Unidos 
para el 2014 reportó un total de 55,1 millones de toneladas de trigo con un rendimiento de 3,0t.ha ${ }^{-1}$ y Canadá alcanzó los 29,2 millones de toneladas con un rendimiento de 3,1 tha $^{-1}$ (FAO, 2017), comparado con Colombia que para la misma fecha produjo solo 16.615 toneladas y un rendimiento que en el mejor de los casos, fue de, 2,3t.ha-1 (MADR, 2017).

Ante este panorama, como indica Burbano y Ortega (2015), el Gobierno Nacional intentó incentivar la constitución de grupos asociativos de agricultores para que se incrementara la capacidad de producción, sin embargo, estas sociedades para poder ser reconocidas e incluidas dentro de las políticas de ayuda, debían cosechar un mínimo de 120 toneladas del cereal al año. En la mayoría de regiones del país, éste proceso presentó grandes retos y dificultades.

Las perspectivas del trigo a futuro, están ligadas a su historia. La dinámica de siembra ha presentado una tendencia decreciente como se evidencia a lo largo de esta revisión.
En la Figura 1 se observa que los años con mayor área sembrada en el país fueron 1955 y 1993. Después de ésta última fecha, el desplome del cultivo fue súbito y constante, lo cual ha coincidido con factores como la apertura económica, los TLC, y recientemente, con la variación en el precio del petróleo y del dólar, lo cual afecta los costos de plaguicidas y fertilizantes usados en cultivo.

Contrario a la producción nacional, las importaciones tienden a incrementarse en el tiempo (Figura 2.). El análisis a las estadísticas de FENALCE (2017) proyectan que desde 1950, anualmente se incrementa la compra de 32.129t $\left(R^{2}=0,95\right)$ llegando a su punto más alto en el 2016, sin la esperanza de un cambio de panorama. Las predicciones de expertos en el cultivo que analizan la producción nacional frente a la importación, concluyen que este sistema agrícola necesita un impulso contundente para evitar a futuro su desaparición (Pareja y Méndez, 2006; Burbano y Ortega, 2015).

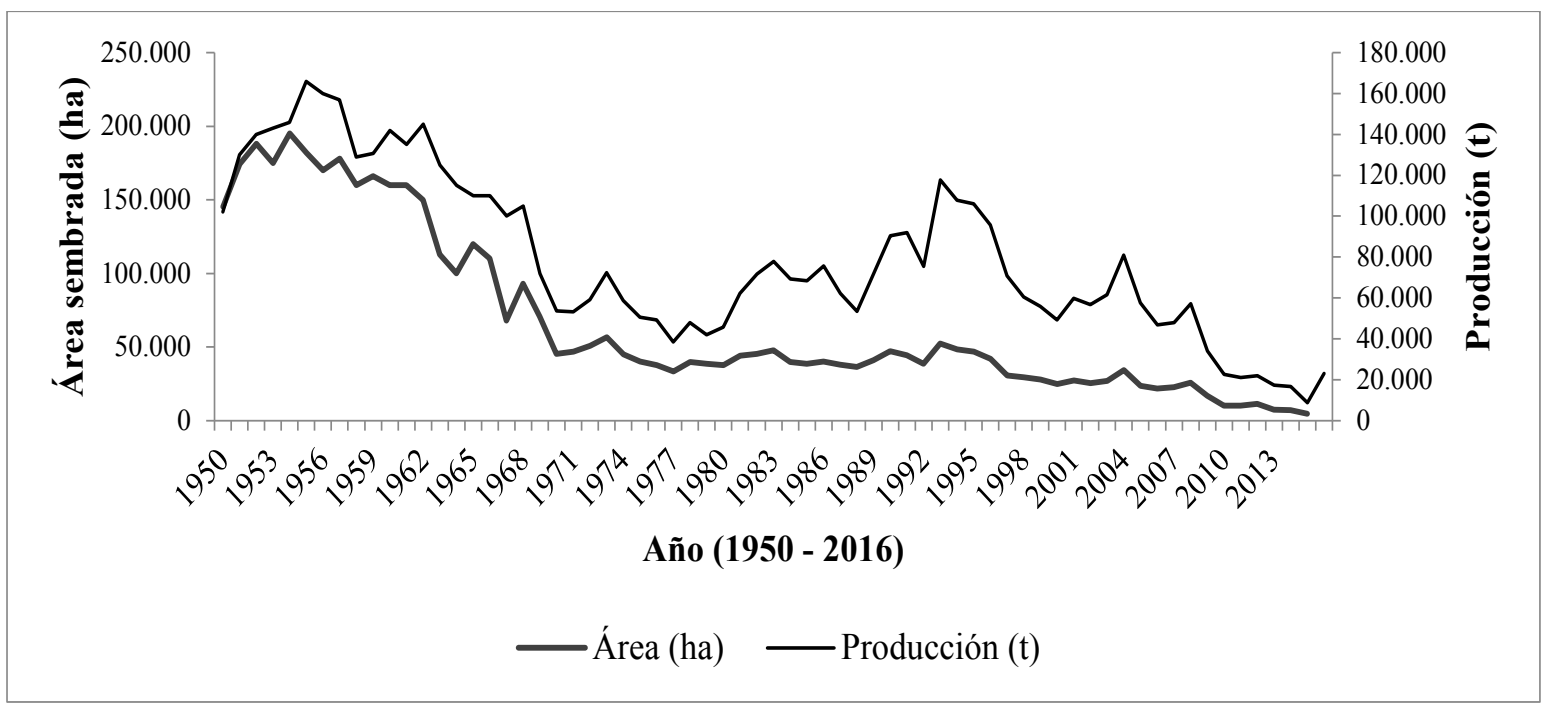

Figura 1. Superficie sembrada y producción de trigo en Colombia $(1950$ - 2016)*.

*Fuente: Valderrama (1976), FENALCE (2017). 


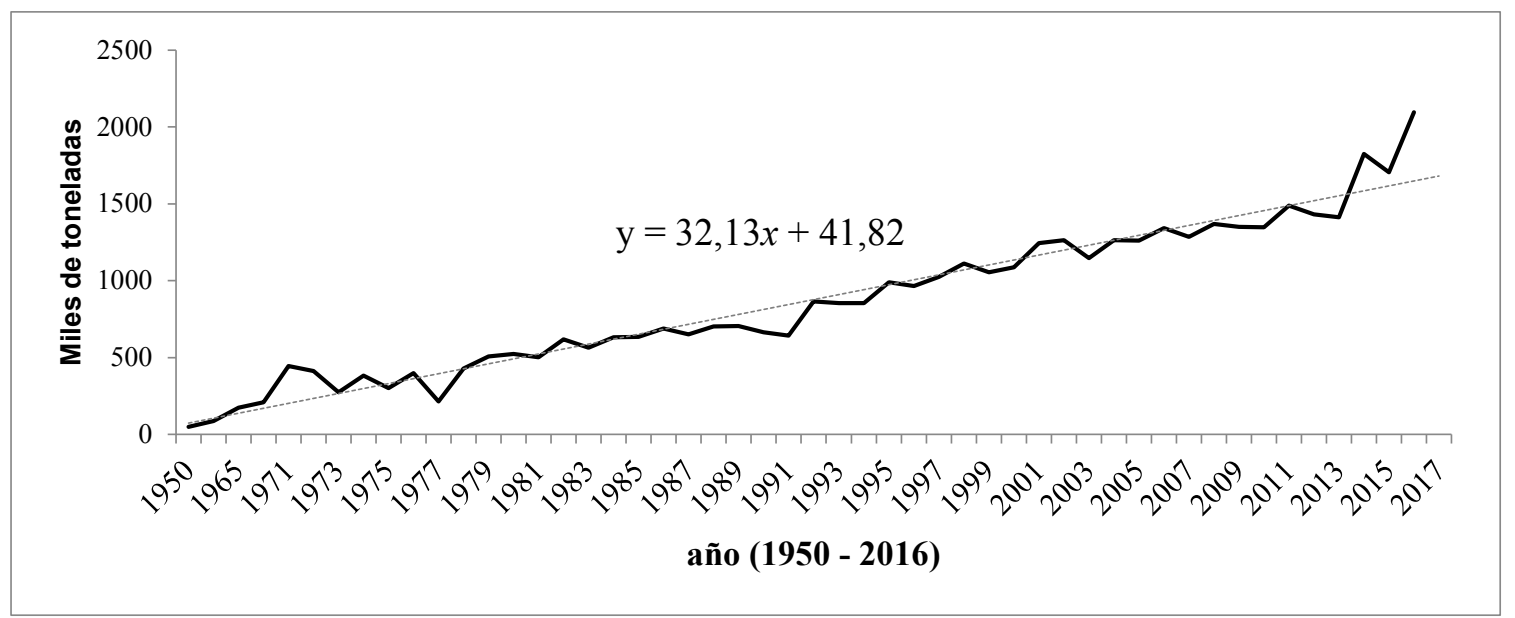

Figura 2. Miles de toneladas importadas de trigo en Colombia (1950 - 2016)*.

*Fuente: FENALCE (2017).

El cambio de una actividad productiva que se ha realizado históricamente y que se encuentra arraigada a la cultura de una región, tiene un tránsito largo y difícil. El insuficiente respaldo a los trigueros, por parte del gobierno, generó inseguridad e intranquilidad en la comunidad campesina. Hizo falta acompañamiento en los procesos de cambio, para que las alternativas generaran bienestar a las familias y de esta manera, hubiese un desarrollo rural adecuado en las zonas históricamente productoras de trigo en los departamentos de Nariño, Cundinamarca y Boyacá (Holt-Giménez et al., 2007; Pareja y Méndez, 2006; Burbano y Ortega, 2015). Con la pérdida del trigo, se disminuye la capacidad de rotación agrícola, la diversidad de productos de la finca, la utilización de subproductos como el tamo para la manufactura de materiales de construcción (ladrillos y muros), artesanías, entre otros.

El impacto social generado en los agricultores debido a la inestabilidad de un cultivo asociado a la macroeconomía, fomentó la migración a las ciudades (Checa, 1997). La sustitución de las actividades agrícolas por las ganaderas, o el reemplazo de los cultivos de trigo por otros (Burbano y Ortega, 2015); tal es el caso de Nariño, donde entidades como FENALCE, CORPOICA y CORPOCEBADA promovieron la conversión de las áreas trigueras al cultivo de Arveja (Checa, 1997).

Actualmente, el trigo se rehúsa a desaparecer, las 6.195ha reportadas para el 2016 por FENALCE (2017) buscan satisfacer nichos de mercado especializado como el de repostería, pastelería y mejoramiento de la calidad harinera. Estos nuevos requerimientos de la industria, sumados a características de mejoramiento agronómico como mayor producción dirigida a zonas agroecológicas específicas y resistencia a enfermedades originan que en el año 2007, se libere por parte de la Universidad de Nariño la variedad FACIANAR-Promesa, y en los años 2014 y 2015 las variedades FNC-Fénix, FNC-Galeras y FNC-Proveedor por parte de FENALCE (ICA, 2014a, 2014b; Sañudo et al., 2015). Este último grupo de variedades, está generando especialmente en el sur de Colombia un nuevo interés en el cereal. 
Bajo el anterior panorama, es necesario desarrollar interrogantes como ¿el trigo en Colombia desaparecerá por la presión constante de importaciones?, ¿es rentable rescatar a un sector agrícola en agonía?, ¿qué pasó con las familias trigueras que cambiaron su vocación agrícola?.

\section{CONCLUSIONES}

El cultivo de trigo en Colombia ha sido afectado por diferentes coyunturas macroeconómicas que inciden directamente en las bases de la producción nacional. El área sembrada y la producción que el país reporta, reflejan las consecuencias de las crisis que ha soportado este sector productivo en los últimos años.

El área sembrada de trigo en el país desde 1993 presenta una tendencia decreciente que pone en duda la sostenibilidad de este sector productivo a futuro. El recorrido histórico en el cual se desarrolló esta revisión muestra un reiterado descuido por parte del Gobierno Nacional en torno a los agricultores que cultivan este cereal.

\section{REFERENCIAS BIBLIOGRÁFICAS}

1. Adams, D.; Mancini, S. 1964. Posibilidades de ajustes en la producción de trigo y los productos competitivos de clima frío. Revista Facultad Nacional de Agronomía, Medellín. 24(61):1 - 26.

2. Afanador, J. 2008. Reseña de "The Political Economy of Food: The Rise and Fall of the Postwar International Food Orde" de H. Friedmann. Revista de Estudios Sociales. 29:180 - 181.
3. Amaguaña, J.; Fuente, D.; Arteaga, G.; Viveros, M. 1995. Respuesta del trigo ICAYacuanquer (Triticum aestivum L.) a la fertilización edáfica y foliar en un sueño del municipio de Pasto. Revista de Ciencias Agrícolas. 13(21):21 - 35.

4. Bejarano, J. 1989. La economía colombiana entre 1922 y 1929, pp. 160-163.

5. Ocampo, J. Historia económica de Colombia. Segunda edición. Siglo veintiuno editores. Bogotá, Colombia. 178p.

6. Bolaños, A.; Campuzano, L.; Merchancano, J.; Coca, A.; Brito, R. 1991. ICA - Gualmatan; variedad mejorada de trigo de excelente calidad galletera para Nariño. En: https://goo.gl/Ld8fvP; consulta: mayo, 2017.

7. Bolaños, A.; Merchancano, J.; Arcila, M.; Yepes, D. 1997. Obonuco sureño 97: nueva variedad mejorada de trigo para zonas de modernización del cultivo en el departamento de Nariño. En: http:// agris.fao.org/agris-search/search. do? recordID=CO2001000335; consulta: abril, 2017.

8. Bolaños, A.; Merchancano, J.; Brito, R.; Fajardo, A. 1993. ICA-Achalay, nueva variedad mejorada de trigo para el departamento de Nariño. ICA Informa. 27(1):64 - 5.

9. Brito, R.; Coca, A.; Sierra, D. 1993. ICA Hunza, variedad mejorada de trigo para el altiplano cundiboyancense. En: http://agris.fao.org/agris-search/search. do? recordID=C01999000164; consulta: abril, 2017.

10. Brito, R.; Coca, A.; Sierra, D.; Delgado, J. 1991. Bochica, variedad mejorada de trigo de altos rendimientos para al altiplano cundiboyacense. En: http:// agris.fao.org/agris-search/search. do? recordID $=$ C01999000105; consulta: abril, 2017. 
11. Burbano, L.: Ortega, C. 2015. El tratado de libre comercio en los discursos de los cultivadores de trigo en Yacuanquer-Nariño. Tendencias. 16(1):125 - 146. doi: http:// dx.doi.org/10.22267/rtend.151601.36.

12. Castro, E. 1980. Variedades de trigo y cebada en el departamento de Nariño. Primera edición. ICA. Colombia. 55 p.

13. Castro, E.; Coca, A.; Sierra, J.; Fajardo, L. 1991. Ica - Tenza; Variedad mejorada de trigo adaptada al altiplano cundiboyacense. En: https://goo.gl/12oAt5; consulta: mayo, 2017.

14. Checa, 0. 1997. Las leguminosas como alternativa de sustitución en zonas de reconversión triguera y cebadera de Nariño. Revista de Ciencias Agrícolas. 15(1y2):88 $-94$.

15. DANE-Departamento Administrativo Nacional de Estadística. 1974. Censo Nacional Agropecuario. DANE. Bogotá, Colombia. 398 p.

16. DANE- Departamento Administrativo Nacional de Estadística. 2011. Matriz de empleo en la base 2005 de las cuentas nacionales. DANE. Bogotá-Colombia. 34 p.

17. Espinal, C., Martínez, H., Prieto, L. 2006. La Cadena del trigo en Colombia, Documento de trabajo No. 126. En: http: www.agrocadenas.gov.co; consulta: mayo, 2017.

18. FAO-Organización de las Naciones Unidas para la alimentación y la agricultura. 2017. Estadísticas de producción de cultivos. En: http://www.fao.org/faostat/ en/\#data/QC; consulta: mayo, 2017.

19. FENALCE- Federación Nacional de Cultivadores de Cereales. 2010. El trigo. En: https://goo.gl/cNfXHk; consulta: mayo, 2017.

20. FENALCE -Federación Nacional de Cultivadores de Cereales. 2017. Índice Cerealista, En: https://goo.gl/1WVfQ5; consulta: mayo, 2017.
21. Holt-Giménez, E.; Altieri, M.; Rosset, P. 2007. Rockefeller y Bill Gates No resolverán los problemas de pobreza y hambre en África. En: https://goo.gl/oG4yg9; consulta: agosto, 2016.

22. ICA. Instituto Colombiano Agropecuario. 2014a. Resolución 001267. Registro de cultivar comercial para producción y comercialización de semillas en Colombia. ICA. Bogotá, Colombia. 6p.

23. ICA. Instituto Colombiano Agropecuario. 2014b. Resolución 001268. Registro de cultivar comercial para producción y comercialización de semillas en Colombia. ICA. Bogotá, Colombia. 6p.

24. IICA. Instituto Interamericano de Cooperación para la Agricultura. 1967. Las ciencias agrícolas en América Latina. IICA. Costa Rica. 656p.

25. Londoño, C. 1998. La apertura económica en Colombia. Pensamiento Humanista. 4: $39-51$.

26. Mac Key, J. 2005. Wheat: Its concept, evolution, and taxonomy, pp: 3-61. En: C. Royo, M. Nachit, N. Difonzo, J. Araus, W. Pfeiffer, G. Slafer, Durum wheat breeding: Current approaches and future strategies. Primera edición. The Haworth Press, E.E.U.U.

27. Machado, A. 2009. La reforma rural, una deuda social y política (No. 1). En: https:// goo.gl/dBHmVM, consulta: agosto, 2016.

28. MADR. Ministerio de agricultura y desarrollo rural. 2017. Producción Nacional por Producto. En: https://goo.gl/QcmbdP, consulta: abril, 2017.

29. Merchancano, J.; Bolaños, A. 1996. Obonuco Sequía-96: Nueva variedad mejorada de trigo para zonas de baja fertilidad y sequía del departamento de Nariño. En: https://goo.gl/jM7AT9; consulta: junio, 2017. 
30. Merheg, H. 2005. Colombia en contravía: Un examen crudo y atrevido al modelo económico y al TLC como política comercial. Primera Edición. Oveja Negra. Bogotá Colombia. $401 \mathrm{p}$.

31. MCIT. Ministerio De Comercio Industria Y Turismo, Proexport Colombia, y OFCCOficina Comercial En Canadá. 2012. Abecé del TLC Colombia-Canadá. En: https:// goo.gl/n6SVtP, consulta: abril, 2016.

32. Moreno, A. 2007. La letra menuda del TLC. Primera edición. Gew, Ed. Bogotá. 428 p.

33. Nuñez, J.; Carvajal, J.; Bautista, L. 2013. El TLC con Estados Unidos y su impacto en el sector agropecuario colombiano: Entre esperanzas e incertidumbres. Revista Electrónica de La Facultad de Derecho. 1(1):118 - 133.

34. Pareja, M.; Méndez, D. 2006. Estudio socioeconómico de las familias productoras de trigo en los Departamentos de Nariño, Boyacá y Cundinamarca, correspondiente a las siembras del semestre A de 2005. FENALCE. Bogotá Colombia. 117p.

35. Patiño, V. 1969. Plantas cultivadas y animales domésticos en América Equinoccial (IV). Imprenta Departamental. Colombia. 503p.

36. Rincón, O., Suarez, A., Contreras, RB., Castiblanco, L., Y Miranda, A., 1981. El cultivo de trigo y la cebada. Temas de Orientación Agropecuaria. 150:1 - 103.

37. Ripoll, M. 2014. La Industria Molinera de Trigo en Colombia: El caso del molino tres castillos, 1940-2012. Economía y Región. 8(2):213 - 265.

38. Sañudo, B.; Muriel, J.; Vanegas, H.; Molina, C. 2015. FNC Proveedor, Genotipo de trigo mejorado para ambientes desfavorables en la zona triguera de Nariño. FENALCE. Pasto, Colombia. 20p.
39. Tapia, M. 1940. El cultivo del trigo. Primera edición. Departamento de Investigaciones Agropecuarias. Colombia. 150p.

40. Trujillo, E.; Torres, E.; Conde, J. 1990. El trigo en la época colonial: Técnica agrícola, producción, molinos y comercio. Universidad de San Buenaventura y Federación Nacional de Molineros de Trigo. Colombia. 108p.

41. Valderrama, M. 1976. El trigo en Colombia. Primera Edición. CIMMYT. México D.F. 23p.

42. Viloria, J. 2007. Economía del Departamento de Nariño: Ruralidad y aislamiento geográfico (No. 87). Centro de Estudios Económicos Regionales. Cartagena, Colombia. 88p.

43. Yepes, E. 1948. Investigaciones sobre la producción de trigo en México y observaciones sobre su cultivo en Colombia. Revista Facultad Nacional de Agronomía. 8(29 - 30):59 - 78.

44. Zapata, M.; López, R. 1967. Programa Nacional del trigo: El cultivo del trigo. ICA. En: https://goo.gl/3LCxek, consulta: mayo, 2017.

45. Zapata, M.; López, R.; Varela, D. 1974. New improved wheat varieties for Colombia. pp. 6-13. En Memories of Latin American wheat conference. Porto Alegre, Brasil.

46. Zarama, M. 1999. El trigo en nuestra comarca, pp. 500-521. En: Muñoz-Cordero, L. Historia de Pasto. Tercera Edición. Academia Nariñense de Historia. San Juan de Pasto, Colombia. 539p. 